\title{
Free Vibration Analysis of a Thin-Walled Beam with Shear Sensitive Material
}

\author{
K. B. Bozdogan ${ }^{1}$ and D. Ozturk ${ }^{2}$ \\ ${ }^{1}$ Kirklareli University, 39000 Kirklareli, Turkey \\ ${ }^{2}$ Ege University, 35000 İmir, Turkey \\ Correspondence should be addressed to K. B. Bozdogan; kbbozdogan@yahoo.com.tr
}

Received 25 June 2013; Accepted 27 August 2013

Academic Editor: Usik Lee

Copyright ( 2013 K. B. Bozdogan and D. Ozturk. This is an open access article distributed under the Creative Commons Attribution License, which permits unrestricted use, distribution, and reproduction in any medium, provided the original work is properly cited.

\begin{abstract}
This paper presents a method for a free vibration analysis of a thin-walled beam of doubly asymmetric cross section filled with shear sensitive material. In the study, first of all, a dynamic transfer matrix method was obtained for planar shear flexure and torsional motion. Then, uncoupled angular frequencies were obtained by using dynamic element transfer matrices and boundary conditions. Coupled frequencies were obtained by the well-known two-dimensional approaches. At the end of the study, a sample taken from the literature was solved, and the results were evaluated in order to test the convenience of the method.
\end{abstract}

\section{Introduction}

In the last two decades research on the dynamics of beams has grown enormously. There are numerous studies [1-29] on the bending-torsion coupled beam. In the beams, the elastic center and the center of mass are not coincident, so the translational and torsional modes are inherently coupled as a result of this offset. Rafezy and Howson [24] proposed an exact dynamic stiffness matrix approach for the three-dimensional, bimaterial beam of doubly asymmetric cross-section. The beam comprises a thin-walled outer layer that encloses and works compositely with its shear sensitive core material.

A dynamic transfer matrix method for the free vibration analysis of a thin-walled beam of doubly asymmetric crosssection filled with shear sensitive materials is suggested in this study. The following assumptions are made in this study: the behaviour of the material is linear elastic, small displacement theory is valid, and the dynamic coupling effect of structure caused by the eccentricity between the center of shear rigidity and the flexural rigidity center is ignored in analysis.

\section{Analysis}

2.1. Physical Model. Figure 1 shows a uniform, three dimensional beam of length $L$. It has a doubly asymmetric cross-section comprising a thin-walled outer layer that encloses shear sensitive material [24]. The outer layer may have the form of an open or closed section that is assumed to provide warping and Saint-Venant rigidity, while the core materials provide Saint-Venant and shear rigidity. These assumptions lead to a model in which a typical cross-section has independent centers of flexure, shear, and mass denoted by $O, S$, and $C$, respectively [24]. For convenience, the origin of the coordinate system is located at the centre of flexure. $O$ gives the result that the axis of elastic flexure coincides with the $z$-axis of the member. The $x$ - and $y$-axes are subsequently aligned with the principle axes of the cross-section. The locations of the points $S$ and $C$ in the coordinate system Oxy are given by $S\left(x_{s}, y_{s}\right)$ and $C\left(x_{c}, y_{c}\right)$, respectively. The resulting elastic shear and mass axes then run parallel to the $z$-axis through $\left(x_{s}, y_{s}\right)$ and $\left(x_{c}, y_{c}\right)$ respectively. When the elastic axis of the beam does not coincide, the lateral and torsional motion of the beam will always be coupled in one or more planes [24].

2.2. Element Transfer Matrices for Planar Motion. The governing equations for $i$ th element of uncoupled thin-walled shear sensitive beam can be written as

$$
\rho_{i} \frac{\partial^{2} U_{i}\left(z_{i}, t\right)}{\partial t^{2}}+E I_{x i} \frac{\partial^{4} U_{i}\left(z_{i}, t\right)}{\partial z_{i}^{4}}-(G A)_{x i} \frac{\partial^{2} U_{i}\left(z_{i}, t\right)}{\partial z_{i}^{2}}=0,
$$




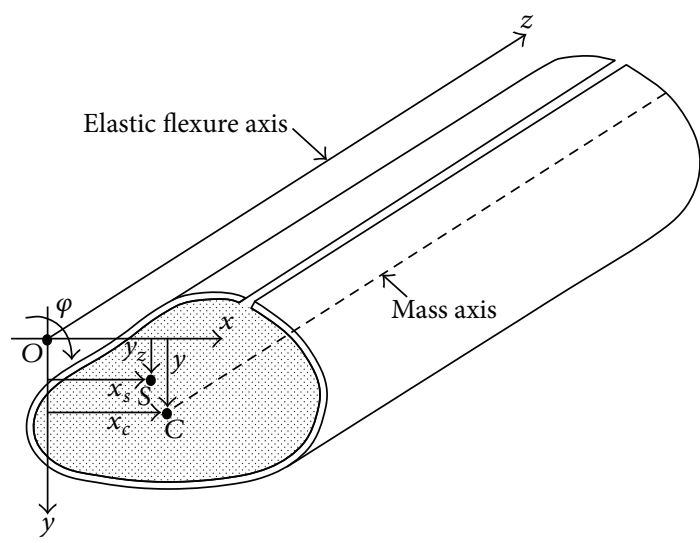

(a)

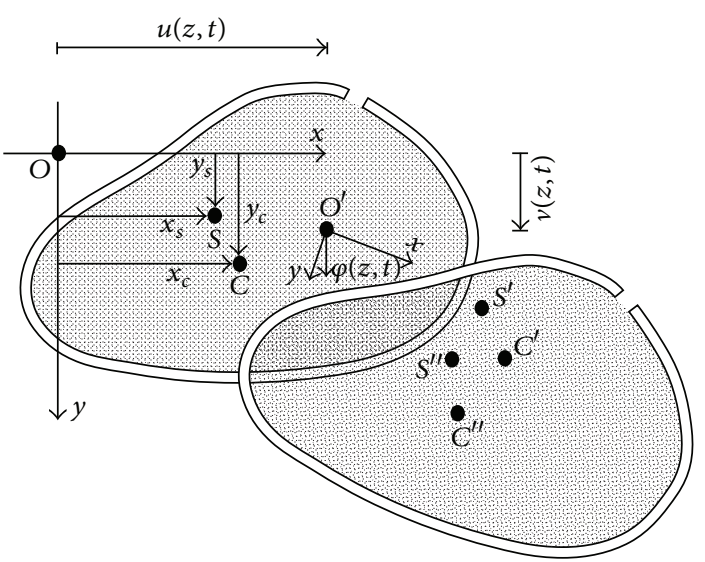

(b)

Figure 1: Typical thin-walled beam [24].

$$
\begin{gathered}
\rho_{i} \frac{\partial^{2} V_{i}\left(z_{i}, t\right)}{\partial t^{2}}+E I_{y i} \frac{\partial^{4} V_{i}\left(z_{i}, t\right)}{\partial z_{i}^{4}}-(G A)_{y i} \frac{\partial^{2} V_{i}\left(z_{i}, t\right)}{\partial z_{i}^{2}}=0, \\
\rho_{i} r_{m}^{2} \frac{\partial^{2} \Psi_{i}\left(z_{i}, t\right)}{\partial t^{2}}+E I_{w i} \frac{\partial^{4} \Psi_{i}\left(z_{i}, t\right)}{\partial z_{i}^{4}}-(G J)_{o i} \frac{\partial^{2} \Psi_{i}\left(z_{i}, t\right)}{\partial z_{i}^{2}}=0,
\end{gathered}
$$

where $G J_{o i}=G_{t i} J_{t i}+G J_{c i}$.

$E I_{x i}$ and $E I_{y i}$ are the flexural rigidity of the $i$ th segment in the $x-z$ and $y-z$ planes, respectively, and $G_{t i} J_{t i}$ and $E I_{w i}$ are the Saint-Venant and warping torsion rigidity of the $i$ th segment about $O$, where $I_{w}$ is the warping moment of inertia or warping constant. $G A_{x i}$ and $G A_{y i}$ are the effective shear rigidities of the core material of the $i$ th segment in $x$ and $y$ directions, respectively, and $G J_{o i}$ is the Saint-Venant torsional rigidity of the core material about $O . \rho_{i}$ are the mass per unit length of the $i$ th segment, and $r_{m}$ is the polar mass radius of gyration of cross section [24].

If a sinusoidal variation of $U, V$, and $\psi$ with circular frequency $\omega$ is assumed then

$$
\begin{aligned}
& U_{i}\left(z_{i}, t\right)=u_{i} \sin \left(\omega_{x} t\right), \\
& V_{i}\left(z_{i}, t\right)=v_{i} \sin \left(\omega_{y} t\right), \\
& \psi_{i}\left(z_{i}, t\right)=\theta_{i} \sin \left(\omega_{\theta} t\right),
\end{aligned}
$$

where $u_{i}, v_{i}$, and $\theta_{i}$ are the amplitudes of the sinusoidally varying displacement.

Substituting (2) in (1) results are

$$
\begin{aligned}
& \frac{d^{4} u_{i}}{d z_{i}^{4}}-\frac{(G A)_{x i}}{(E I)_{x i}} \frac{d^{2} u_{i}}{d z_{i}^{2}}-\frac{p_{i}}{(E I)_{x i}} \omega_{x}^{2} u_{i}=0, \\
& \frac{d^{4} v_{i}}{d z_{i}^{4}}-\frac{(G A)_{y i}}{(E I)_{y i}} \frac{d^{2} v_{i}}{d z_{i}^{2}}-\frac{p_{i}}{(E I)_{y i}} \omega_{y}^{2} v_{i}=0, \\
& \frac{d^{4} \theta_{i}}{d z_{i}^{4}}-\frac{(G J)_{o i}}{(E I)_{w i}} \frac{d^{2} \theta_{i}}{d \theta_{i}^{2}}-\frac{p_{i} r_{m}^{2}}{(E I)_{w i}} \omega_{\theta}^{2} \theta_{i}=0 .
\end{aligned}
$$

When (3) is solved with respect to $z_{i}, u_{i}\left(z_{i}\right), v_{i}\left(z_{i}\right)$, and $\theta_{i}\left(z_{i}\right)$ can be obtained as follows:

$$
\begin{aligned}
u_{i}\left(z_{i}\right)= & c_{1} \cosh \left(a_{x i} z_{i}\right)+c_{2} \sinh \left(a_{x i} z_{i}\right) \\
& +c_{3} \cos \left(b_{x i} z_{i}\right)+c_{4} \sin \left(b_{x i} z_{i}\right), \\
v_{i}\left(z_{i}\right)= & c_{5} \cosh \left(a_{y i} z_{i}\right)+c_{6} \sinh \left(a_{y i} z_{i}\right) \\
& +c_{7} \cos \left(b_{y i} z_{i}\right)+c_{8} \sin \left(b_{y i} z_{i}\right), \\
\theta_{i}\left(z_{i}\right)= & c_{9} \cosh \left(a_{\theta i} z_{i}\right)+c_{10} \sinh \left(a_{\theta i} z_{i}\right) \\
& +c_{11} \cos \left(b_{\theta i} z_{i}\right)+c_{12} \sin \left(b_{\theta i} z_{i}\right),
\end{aligned}
$$

where $\alpha_{x i}, \alpha_{y i}, \alpha_{\theta i}, b_{x i}, b_{y i}$, and $b_{\theta i}$ can be calculated as follows:

$$
\begin{gathered}
a_{x i}=\sqrt{\frac{s_{x i}+p_{x i}}{2}}, \quad a_{y i}=\sqrt{\frac{s_{y i}+p_{y i}}{2},} \\
a_{\theta i}=\sqrt{\frac{s_{\theta i}+p_{\theta i}}{2}}, \quad b_{x i}=\sqrt{\frac{-s_{x i}+p_{x i}}{2}}, \\
b_{y i}=\sqrt{\frac{-s_{y i}+p_{y i}}{2}}, \quad b_{\theta i}=\sqrt{\frac{-s_{\theta i}+p_{\theta i}}{2}}, \\
p_{x i}=\sqrt{\left(\frac{G A_{x i}}{E I_{x i}}\right)^{2}+4 * \frac{p_{i} \omega_{x}^{2}}{(E I)_{x i}}}, \\
p_{y i}=\sqrt{\left(\frac{G A_{y i}}{E I_{y i}}\right)^{2}+4 * \frac{p_{i} \omega_{y}^{2}}{(E I)_{y i}}},
\end{gathered}
$$




$$
\begin{gathered}
p_{\theta i}=\sqrt{\left(\frac{G J_{o i}}{E I_{w i}}\right)^{2}+4 * \frac{p_{i} \omega_{\theta}^{2}}{(E I)_{w i}}}, \\
s_{x i}=\frac{(G A)_{x i}}{(E I)_{x i}}, \quad s_{y i}=\frac{(G A)_{y i}}{(E I)_{y i}}, \quad s_{\theta i}=\frac{(G J)_{o i}}{(E I)_{w i}} .
\end{gathered}
$$

By using (4), (5), and (6), the rotation angles in $x$ and $y$ directions $\left(u_{i}^{\prime}, v_{i}^{\prime}\right)$, rate of twist $\left(\theta_{i}^{\prime}\right)$, bending moments in $x$ and $y$ directions $\left(M_{x i}, M_{y i}\right)$ and bimoment $\left(M_{w i}\right)$, shear forces in $x$ and $y$ directions $\left(V_{x i}, V_{y i}\right)$, and torque $\left(M_{t i}\right)$ for $i$ th element can be obtained as follows:

$$
\begin{aligned}
\frac{d u_{i}\left(z_{i}\right)}{d z_{i}}= & c_{1} a_{x i} \sinh \left(a_{x i} z_{i}\right)+c_{2} a_{x i} \cosh \left(a_{x i} z_{i}\right) \\
& -c_{3} b_{x i} \sin \left(b_{x i} z_{i}\right)+c_{4} b_{x i} \cos \left(b_{x i} z_{i}\right) \\
\frac{d v_{i}\left(z_{i}\right)}{d z_{i}}= & c_{5} a_{y i} \sinh \left(a_{y i} z_{i}\right)+c_{6} a_{y i} \cosh \left(a_{y i} z_{i}\right) \\
& -c_{7} b_{y i} \sin \left(b_{y i} z_{i}\right)+c_{8} b_{y i} \cos \left(b_{y i} z_{i}\right)
\end{aligned}
$$$$
\frac{d \theta_{i}\left(z_{i}\right)}{d z_{i}}=c_{9} a_{\theta i} \sinh \left(a_{\theta i} z_{i}\right)+c_{10} a_{\theta i} \cosh \left(a_{\theta i} z_{i}\right)
$$$$
-c_{11} b_{\theta i} \sin \left(b_{\theta i} z_{i}\right)+c_{12} b_{\theta i} \cos \left(b_{\theta i} z_{i}\right),
$$$$
M_{x i}\left(z_{i}\right)=E I_{x i} \frac{d^{2} u_{i}\left(z_{i}\right)}{d z_{i}^{2}}
$$$$
=E I_{x i}\left[c_{1} a_{x i}^{2} \cosh \left(a_{x i} z_{i}\right)+c_{2} a_{x i}^{2} \sinh \left(a_{x i} z_{i}\right)\right.
$$$$
\left.-c_{3} b_{x i}^{2} \cos \left(b_{x i} z_{i}\right)-c_{4} b_{x i}^{2} \sin \left(b_{x i} z_{i}\right)\right],
$$$$
M_{y i}\left(z_{i}\right)=E I_{y i} \frac{d^{2} v_{i}\left(z_{i}\right)}{d z_{i}^{2}}
$$$$
=E I_{y i}\left[c_{5} a_{y i}^{2} \cosh \left(a_{y i} z_{i}\right)+c_{6} a_{y i}^{2} \sinh \left(a_{y i} z_{i}\right)\right.
$$$$
\left.-c_{7} b_{y i}^{2} \cos \left(b_{y i} z_{i}\right)-c_{8} b_{y i}^{2} \sin \left(b_{y i} z_{i}\right)\right],
$$

$$
\begin{aligned}
M_{w i}\left(z_{i}\right)= & E I_{w i} \frac{d^{2} \theta_{i}\left(z_{i}\right)}{d z_{i}^{2}} \\
=E I_{w i} & {\left[c_{9} a_{\theta i}^{2} \cosh \left(a_{\theta i} z_{i}\right)+c_{10} a_{\theta i}^{2} \sinh \left(a_{\theta i} z_{i}\right)\right.} \\
& \left.\quad-c_{11} b_{\theta i}^{2} \cos \left(b_{\theta i} z_{i}\right)-c_{12} b_{\theta i}^{2} \sin \left(b_{\theta i} z_{i}\right)\right],
\end{aligned}
$$

$$
\begin{aligned}
V_{x i}\left(z_{i}\right)= & E I_{x i} \frac{d^{3} u_{i}\left(z_{i}\right)}{d z_{i}^{3}}-(G A)_{x i} \frac{d u_{i}\left(z_{i}\right)}{d z_{i}} \\
= & {\left[E I_{x i} a_{x i}^{3} \sinh \left(a_{x i} z_{i}\right)-(G A)_{x i} a_{x i} \sinh \left(a_{x i} z_{i}\right)\right] c_{1} } \\
+ & {\left[E I_{x i} a_{x i}^{3} \cosh \left(a_{x i} z_{i}\right)\right.} \\
& \left.\quad-(G A)_{x i} a_{x i} \cosh \left(a_{x i} z_{i}\right)\right] c_{2} \\
+ & {\left[(E I)_{x i} b_{x i}^{3} \sin \left(b_{x i} z_{i}\right)+(G A)_{x i} b_{x i} \sin \left(b_{x i} z_{i}\right)\right] c_{3} } \\
+ & {\left[-(E I)_{x i} b_{x i}^{3} \cos \left(b_{x i} z_{i}\right)\right.} \\
& \left.-(G A)_{x i} b_{x i} \cos \left(b_{x i} z_{i}\right)\right] c_{4},
\end{aligned}
$$

$$
\begin{aligned}
V_{y i}\left(z_{i}\right)= & E I_{y i} \frac{d^{3} v_{i}\left(z_{i}\right)}{d z_{i}^{3}}-(G A)_{y i} \frac{d v_{i}\left(z_{i}\right)}{d z_{i}} \\
= & {\left[E I_{y i} a_{y i}^{3} \sinh \left(a_{y i} z_{i}\right)-(G A)_{y i} a_{y i} \sinh \left(a_{y i} z_{i}\right)\right] c_{5} } \\
+ & {\left[E I_{y i} a_{y i}^{3} \cosh \left(a_{y i} z_{i}\right)\right.} \\
& \left.-(G A)_{y i} a_{y i} \cosh \left(a_{y i} z_{i}\right)\right] c_{6} \\
+ & {\left[(E I)_{y i} b_{y i}^{3} \sin \left(b_{y i} z_{i}\right)+(G A)_{y i} b_{y i} \sin \left(b_{y i} z_{i}\right)\right] c_{7} } \\
+ & {\left[-(E I)_{y i} b_{y i}^{3} \cos \left(b_{y i} z_{i}\right)\right.} \\
& \left.\quad-(G A)_{y i} b_{y i} \cos \left(b_{y i} z_{i}\right)\right] c_{8},
\end{aligned}
$$$$
M_{t i}\left(z_{i}\right)=E I_{w i} \frac{d^{3} \theta_{i}\left(z_{i}\right)}{d z_{i}^{3}}-(G J)_{o i} \frac{d \theta_{i}\left(z_{i}\right)}{d z_{i}}
$$$$
=\left[E I_{\theta i} a_{\theta i}^{3} \sinh \left(a_{\theta i} z_{i}\right)-(G J)_{o i} a_{\theta i} \sinh \left(a_{\theta i} z_{i}\right)\right] c_{9}
$$$$
+\left[E I_{w i} a_{\theta i}^{3} \cosh \left(a_{\theta i} z_{i}\right)\right.
$$$$
\left.-(G J)_{i o} a_{\theta i} \cosh \left(a_{\theta i} z_{i}\right)\right] c_{10}
$$$$
+\left[(E I)_{w i} b_{\theta i}^{3} \sin \left(b_{\theta i} z_{i}\right)+(G J)_{o i} b_{\theta i} \sin \left(b_{\theta i} z_{i}\right)\right] c_{11}
$$$$
+\left[-(E I)_{w i} b_{\theta i}^{3} \cos \left(b_{\theta i} z_{i}\right)\right.
$$$$
\left.-(G J)_{o i} b_{y i} \cos \left(b_{\theta i} z_{i}\right)\right] c_{12} \text {. }
$$

The following equation shows the matrix form of $(4),(8),(11)$, and (14):

$$
\left[\begin{array}{c}
u_{i}\left(z_{i}\right) \\
u_{i}^{\prime}\left(z_{i}\right) \\
M_{x i}\left(z_{i}\right) \\
V_{x i}\left(z_{i}\right)
\end{array}\right]=A_{x i}\left(z_{i}\right)\left[\begin{array}{c}
c_{1} \\
c_{2} \\
c_{3} \\
c_{4}
\end{array}\right] .
$$


For the $y$ direction, the following shows the matrix form of (5), (9), (12), and (15):

$$
\left[\begin{array}{c}
v_{i}\left(z_{i}\right) \\
v_{i}^{\prime}\left(z_{i}\right) \\
M_{y i}\left(z_{i}\right) \\
V_{y i}\left(z_{i}\right)
\end{array}\right]=A_{y i}\left(z_{i}\right)\left[\begin{array}{c}
c_{5} \\
c_{6} \\
c_{7} \\
c_{8}
\end{array}\right] .
$$

Similarly, torsional motion can be written:

$$
\left[\begin{array}{c}
\theta_{i}\left(z_{i}\right) \\
\theta_{i}^{\prime}\left(z_{i}\right) \\
M_{w i}\left(z_{i}\right) \\
M_{t i}\left(z_{i}\right)
\end{array}\right]=A_{\theta i}\left(z_{i}\right)\left[\begin{array}{c}
c_{9} \\
c_{10} \\
c_{11} \\
c_{12}
\end{array}\right] .
$$

At the initial point of the $i$ th element, (17), (18), and (19) can be written as follows:

$$
\begin{gathered}
{\left[\begin{array}{c}
u_{i}(0) \\
u_{i}^{\prime}(0) \\
M_{x i}(0) \\
V_{x i}(0)
\end{array}\right]=A_{x i}(0)\left[\begin{array}{l}
c_{1} \\
c_{2} \\
c_{3} \\
c_{4}
\end{array}\right],} \\
{\left[\begin{array}{c}
v_{i}(0) \\
v_{i}^{\prime}(0) \\
M_{y i}(0) \\
V_{y i}(0)
\end{array}\right]=A_{y i}(0)\left[\begin{array}{l}
c_{5} \\
c_{6} \\
c_{7} \\
c_{8}
\end{array}\right],} \\
{\left[\begin{array}{c}
\theta_{i}(0) \\
\theta_{i}^{\prime}(0) \\
M_{w i}(0) \\
M_{t i}(0)
\end{array}\right]=A_{\theta i}(0)\left[\begin{array}{l}
c_{9} \\
c_{10} \\
c_{11} \\
c_{12}
\end{array}\right] .}
\end{gathered}
$$

When vector $c$ is solved from (20) and is substituted in (17), the following is obtained:

$$
\left[\begin{array}{c}
u_{i}\left(z_{i}\right) \\
u_{i}^{\prime}\left(z_{i}\right) \\
M_{x i}\left(z_{i}\right) \\
V_{x i}\left(z_{i}\right)
\end{array}\right]=A_{x i}\left(z_{i}\right) A_{x i}(0)^{-1}\left[\begin{array}{c}
u_{i}(0) \\
u_{i}^{\prime}(0) \\
M_{x i}(0) \\
V_{x i}(0)
\end{array}\right] .
$$

For $z_{i}=l_{i},(23)$ can be written as

$$
\left[\begin{array}{c}
u_{i}\left(l_{i}\right) \\
u_{i}^{\prime}\left(l_{i}\right) \\
M_{x i}\left(l_{i}\right) \\
V_{x i}\left(l_{i}\right)
\end{array}\right]=T_{x i}\left[\begin{array}{c}
u_{i}(0) \\
u_{i}^{\prime}(0) \\
M_{x i}(0) \\
V_{x i}(0)
\end{array}\right],
$$

where $T_{x i}$ is the element dynamic transfer matrix of the $i$ th element.

For the $y$ direction, (23) and (24) can be written as follows:

$$
\begin{gathered}
{\left[\begin{array}{c}
v_{i}\left(z_{i}\right) \\
v_{i}^{\prime}\left(z_{i}\right) \\
M_{y i}\left(z_{i}\right) \\
V_{y i}\left(z_{i}\right)
\end{array}\right]=A_{y i}\left(z_{i}\right) A_{y i}(0)^{-1}\left[\begin{array}{c}
v_{i}(0) \\
v_{i}^{\prime}(0) \\
M_{y i}(0) \\
V_{y i}(0)
\end{array}\right],} \\
{\left[\begin{array}{c}
v_{i}\left(l_{i}\right) \\
v_{i}^{\prime}\left(l_{i}\right) \\
M_{y i}\left(l_{i}\right) \\
V_{y i}\left(l_{i}\right)
\end{array}\right]=T_{y i}\left[\begin{array}{c}
v_{i}(0) \\
v_{i}^{\prime}(0) \\
M_{y i}(0) \\
V_{y i}(0)
\end{array}\right]}
\end{gathered}
$$

Similarly, rotation motion can be written in equations as follows:

$$
\left[\begin{array}{c}
\theta_{i}\left(z_{i}\right) \\
\theta_{i}^{\prime}\left(z_{i}\right) \\
M_{w i}\left(z_{i}\right) \\
M_{t i}\left(z_{i}\right)
\end{array}\right]=A_{\theta i}\left(z_{i}\right) A_{\theta i}(0)^{-1}\left[\begin{array}{c}
\theta_{i}(0) \\
\theta_{i}^{\prime}(0) \\
M_{w i}(0) \\
M_{t i}(0)
\end{array}\right],
$$

$$
\left[\begin{array}{c}
\theta_{i}\left(l_{i}\right) \\
\theta_{i}^{\prime}\left(l_{i}\right) \\
M_{w i}\left(l_{i}\right) \\
M_{t i}\left(l_{i}\right)
\end{array}\right]=T_{\theta i}\left[\begin{array}{c}
\theta_{i}(0) \\
\theta_{i}^{\prime}(0) \\
M_{w i}(0) \\
M_{t i}(0)
\end{array}\right]
$$

If (24) is written successively, the displacements-internal forces relationship between the initial part and end of the beam - can be found as follows:

$$
\begin{aligned}
{\left[\begin{array}{c}
u_{\text {end }} \\
u_{\text {end }}^{\prime} \\
M_{x \text { nd }} \\
V_{x \text { end }}
\end{array}\right] } & =T_{x n} T_{x(n-1)} \cdots T_{x 2} T_{x 1}\left[\begin{array}{c}
u_{\text {initial }} \\
u_{\text {initial }}^{\prime} \\
M_{x \text { initial }} \\
V_{x \text { initial }}
\end{array}\right] \\
& =t_{x}\left[\begin{array}{c}
u_{\text {initial }} \\
u_{\text {initial }}^{\prime} \\
M_{x \text { initial }} \\
V_{x \text { initial }}
\end{array}\right] .
\end{aligned}
$$

For $y$ and rotation motion, (29) can be written as follows:

$$
\left[\begin{array}{c}
v_{\text {end }} \\
v_{\text {end }}^{\prime} \\
M_{y \text { end }} \\
V_{y \text { end }}
\end{array}\right]=T_{y n} T_{y(n-1)} \cdots T_{y 2} T_{y 1}\left[\begin{array}{c}
v_{\text {initial }} \\
v_{\text {initial }}^{\prime} \\
M_{y \text { initial }} \\
V_{y \text { initial }}
\end{array}\right]
$$

$$
=t_{y}\left[\begin{array}{c}
v_{\text {initial }} \\
v_{\text {initial }}^{\prime} \\
M_{y \text { initial }} \\
V_{y \text { initial }}
\end{array}\right],
$$

$$
\begin{aligned}
{\left[\begin{array}{c}
\theta_{\text {end }} \\
\theta_{\text {end }}^{\prime} \\
M_{\text {wend }} \\
M_{\text {tend }}
\end{array}\right] } & =T_{\theta n} T_{\theta(n-1)} \cdots T_{\theta 2} T_{\theta 1}\left[\begin{array}{c}
\theta_{\text {initial }} \\
\theta_{\text {initial }}^{\prime} \\
M_{\text {winitial }} \\
M_{\text {tinitial }}
\end{array}\right] \\
& =t_{\theta}\left[\begin{array}{c}
\theta_{\text {initial }} \\
\theta_{\text {initial }}^{\prime} \\
M_{\text {winitial }} \\
M_{\text {tinitial }}
\end{array}\right] .
\end{aligned}
$$

The eigenvalue equation for a thin-walled beam filled with shear sensitive material can be established using (29), (30), and the specific boundary conditions are as follows.

(1) Clamped-Free: $f_{x}=t_{x}(3,3)^{*} t_{x}(4,4)-t_{x}(3,4)^{*} t_{x}(4$, 3) $=0, f_{y}=t_{y}(3,3)^{*} t_{y}(4,4)-t_{y}(3,4)^{*} t_{y}(4,3)=$ $0, f_{\theta}=t_{\theta}(3,3)^{*} t_{\theta}(4,4)-t_{\theta}(3,4)^{*} t_{\theta}(4,3)=0$.

(2) Clamped-Clamped: $f_{x}=t_{x}(1,3)^{*} t_{x}(2,4)-t_{x}(1$, $4)^{*} t_{x}(2,3)=0, f_{y}=t_{y}(1,3)^{*} t_{y}(2,4)-t_{y}(1,4)^{*} t_{y}(2$, 3) $=0, f_{\theta}=t_{\theta}(1,3)^{*} t_{\theta}(2,4)-t_{\theta}(1,4)^{*} t_{\theta}(2,3)=0$. 


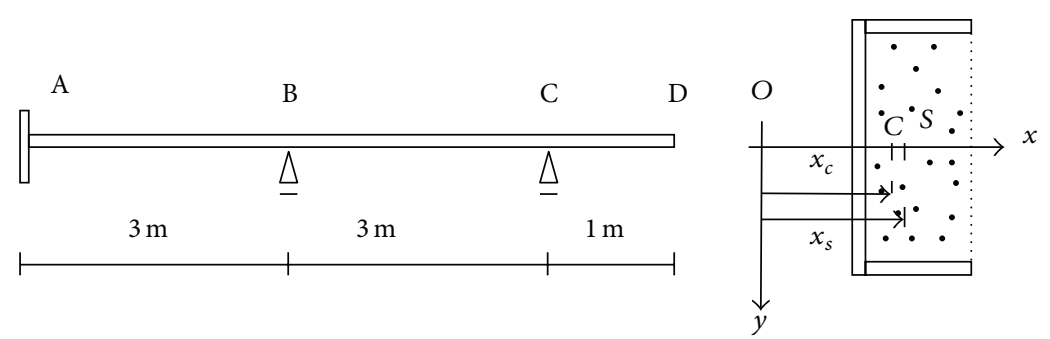

Figure 2: The doubly asymmetric, continuous channel section and the cross section of beam of example 2 with warping allowed at B, C, and $\mathrm{D}$ but fully constrained at A.

(3) Simply-Simply: $f_{x}=t_{x}(1,2)^{*} t_{x}(3,4)-t_{x}(3,2)^{*} t_{x}(1$, $4)=0, f_{y}=t_{y}(1,2)^{*} t_{y}(3,4)-t_{y}(3,2)^{*} t_{y}(1,4)=$ $0, f_{\theta}=t_{\theta}(1,2)^{*} t_{\theta}(3,4)-t_{\theta}(3,2)^{*} t_{\theta}(1,4)=0$.

(4) Free-Free: $f_{x}=t_{x}(3,1)^{*} t_{x}(4,2)-t_{x}(3,2)^{*} t_{x}(4,1)=$ $0, f_{y}=t_{y}(3,1)^{*} t_{y}(4,2)-t_{y}(3,2)^{*} t_{y}(4,1)=0, f_{\theta}=$ $t_{\theta}(3,1)^{*} t_{\theta}(4,2)-t_{\theta}(3,2)^{*} t_{\theta}(4,1)=0$.

(5) Clamped-Simply: $f_{x}=t_{x}(1,3)^{*} t_{x}(3,4)-t_{x}(1$, $4)^{*} t_{x}(3,3)=0, f_{y}=t_{y}(1,3)^{*} t_{y}(3,4)-t_{y}(1,4)^{*} t_{y}(3$, $3)=0, f_{\theta}=t_{\theta}(1,3)^{*} t_{\theta}(3,4)-t_{\theta}(1,4)^{*} t_{\theta}(3,3)=0$.

In frequency equations the values of $\omega$, which set the determinant to zero, are the uncoupled angular frequencies.

2.3. Coupled Frequencies. Ignoring the dynamic coupling effect of structure caused by the eccentricity between the center of shear rigidity and the geometric center the coupled frequencies of the shear torsional beam can be obtained by using uncoupled frequencies and the well-known equation as follows [28]:

$$
\begin{aligned}
& \left|\begin{array}{ccc}
\omega_{j}^{(i)^{2}}-\omega_{x}^{(i)^{2}} & 0 & -y_{c} \omega_{j}^{(i)^{2}} \\
0 & \omega_{j}^{(i)^{2}}-\omega_{y}^{(i)^{2}} & x_{c} \omega_{j}^{(i)^{2}} \\
-y_{c} \omega_{j}^{(i)^{2}} & x_{c} \omega_{j}^{(i)^{2}} & r_{m}^{2}\left(\omega_{j}^{(i)^{2}}-\omega_{\theta}^{(i)^{2}}\right.
\end{array}\right|=0 \\
& (j=1,2,3) \quad(i=1,2,3 \ldots) .
\end{aligned}
$$

\section{Procedure of Computation}

A program that considers the method presented in this study as a basis has been prepared in MATLAB, and the operation stages are presented below.

(1) element dynamic Transfer matrices are calculated for each element by using (24), (26), and (28).

(2) System dynamic transfer matrices (see (29)-(30)) are obtained with the help of element transfer matrices.

(3) The angular frequencies of uncoupled vibrations are obtained by using the boundary conditions.

(4) The coupled angular frequencies are found by using (31).

\section{A Numerical Example}

In this part of the study two numerical examples were solved by a program written in MATLAB to validate the presented method. The results are compared with those given in the literature.

4.1. Numerical Example 1. The first example considers the beam studied by Tanaka and Bercin [11]. A typical uniform thin-walled beam has a length of $1.5 \mathrm{~m}$ with a doubly asymmetric cross section. The properties of the cross section are as follows:

$$
\begin{aligned}
& x_{c}=0.02316, y_{c}=0.02625, \rho=1.947 \mathrm{~kg} / \mathrm{m}, r_{m}^{2}= \\
& 3.0303^{*} 10^{-3} \mathrm{~m}^{2}, \\
& E I_{x}=73480 \mathrm{Nm}^{2}, E I_{y}=16680 \mathrm{Nm}^{2}, E I_{w}= \\
& 23.64 \mathrm{Nm}^{4} \text {, and } G J_{0}=10.81 \mathrm{Nm}^{2} .
\end{aligned}
$$

The first three coupled natural frequencies of the beam are calculated by the presented method and compared with the results by Tanaka and Bercin [11] and Rafezy and Howson [24] in Table 1 for clamped-free (C-F) and simply-simply (SS) boundary conditions.

4.2. Numerical Example 2. A typical continuous beam with a doubly asymmetric cross section is considered in this example (Figure 2).

The beam comprises a thin-walled outer layer and a shear core with the following properties between support points $\mathrm{A}$ and $\mathrm{B}$. The typical uniform thin-walled beam has a length of $1.5 \mathrm{~m}$ with a doubly asymmetric cross section. The properties of the cross section are as follows:

$$
\begin{aligned}
& x_{s}=0.08, y_{s}=0.03, x_{c}=0.05, y_{c}=0.02, \rho= \\
& 20 \mathrm{~kg} / \mathrm{m}, r_{m}^{2}=0.008 \mathrm{~m}^{2}, \\
& E I_{x}=2.16^{*} 10^{6} \mathrm{Nm}^{2}, E I_{y}=1.73^{*} 10^{6} \mathrm{Nm}^{2}, G_{t} J_{t}= \\
& 3200 \mathrm{Nm}^{2}, \\
& E I_{w}=1.4^{*} 10^{3} \mathrm{Nm}^{4}, G A_{x}=600000 \mathrm{~N}, G A_{y}= \\
& 600000 \mathrm{~N}, \text { and } G J_{c}=3800 \mathrm{Nm}^{2} .
\end{aligned}
$$

The shear core is omitted between points B and D, where the cross-sectional properties remain unchanged, except that $G A_{x}=G A_{y}=G J_{c}=0$, and the small change in $\rho$ has been ignored. 
TABLE 1: Coupled natural frequencies for the beam of example 1.

\begin{tabular}{|c|c|c|c|c|c|c|c|c|c|}
\hline \multicolumn{10}{|c|}{ Natural frequencies $(\mathrm{Hz})$} \\
\hline \multirow{2}{*}{$\mathrm{BC}$} & \multicolumn{3}{|c|}{ Proposed method } & \multicolumn{3}{|c|}{ Tanaka and Bercin [11] } & \multicolumn{3}{|c|}{ Rafezy and Howson [24] } \\
\hline & $f_{1}$ & $f_{2}$ & $f_{3}$ & $f_{1}$ & $f_{2}$ & $f_{3}$ & $f_{1}$ & $f_{2}$ & $f_{3}$ \\
\hline $\mathrm{C}-\mathrm{F}$ & 17.17 & 27.31 & 59.10 & 17.03 & 27.58 & 59.25 & 17.17 & 27.31 & 59.10 \\
\hline S-S & 44.71 & 75.14 & 164.87 & 41.48 & 74.12 & 164.11 & 44.71 & 75.14 & 164.87 \\
\hline
\end{tabular}

TABLE 2: Coupled natural frequencies of the continuous beam of example 2 .

\begin{tabular}{lccc}
\hline $\begin{array}{l}\text { Frequency } \\
\text { number }\end{array}$ & This study & Rafezy and Howson[24] & Difference (\%) \\
\hline 1 & 6.906 & 6.940 & -0.49 \\
2 & 19.763 & 19.796 & -0.17 \\
3 & 35.461 & 33.836 & 4.80 \\
\hline
\end{tabular}

The first three coupled natural frequencies of the beam are calculated by the presented method and compared with the results of Rafezy and Howson [24] in Table 2.

The main source of error between the proposed method and Rafezy and Howson methods is the eccentricity between the center of shear stiffness and flexural stiffness which was not taken into account in the proposed method.

\section{Conclusions}

This paper presents a method for a free vibration analysis of a thin-walled beam of doubly asymmetric cross section filled with shear sensitive material. In the study, first of all, a dynamic transfer matrix method was obtained for planar shear flexure and torsional motion. Then, uncoupled angular frequencies were obtained by using dynamic element transfer matrices and boundary conditions. Coupled frequencies were obtained by the well-known two-dimensional approaches. It was observed from the sample taken from the literature that the presented method gave sufficient results. The error margin of the proposed method is shown to be less than 5\%. The main source of error is the eccentricity between the center of shear stiffness and flexural stiffness which was not taken into account in the proposed method.

The transfer matrix method is an efficient and computerized method which also provides a fast and practical solution since the dimension of the matrix for the elements and system never changes. Because of this the proposed method is simple and accurate enough to be used both at the concept design stage and for final analyses.

\section{References}

[1] F. Y. Cheng, "Vibrations of timoshenko beams and frameworks," Journal of Structural Engineering, vol. 96, no. 3, pp. 551-571, 1970.

[2] C. Mei, "Coupled vibrations of thin-walled beams of open section using the finite element method," International Journal of Mechanical Sciences, vol. 12, no. 10, pp. 883-891, 1970.

[3] W. L. Hallauer and R. Y. L. Liu, "Beam bending-torsion dynamic stiffness method for calculation of exact vibration modes,"
Journal of Sound and Vibration, vol. 85, no. 1, pp. 105-113, 1982.

[4] E. Dokumaci, "An exact solution for coupled bending and torsion vibrations of uniform beams having single cross-sectional symmetry," Journal of Sound and Vibration, vol. 119, no. 3, pp. 443-449, 1987.

[5] R. H. Gutierrez and P. A. A. Laura, "Approximate analysis of coupled flexural-torsional vibrations of a beam of non-uniform cross-section using the optimized rayleigh method," Journal of Sound and Vibration, vol. 114, no. 2, pp. 393-397, 1987.

[6] J. R. Banerjee, "Coupled bending-torsional dynamic stiffness matrix for beam elements," International Journal for Numerical Methods in Engineering, vol. 28, no. 6, pp. 1283-1298, 1989.

[7] J. R. Banerjee and F. W. Williams, "Coupled bending-torsional dynamic stiffness matrix for timoshenko beam elements," Computers and Structures, vol. 42, no. 3, pp. 301-310, 1992.

[8] J. R. Banerjee and F. W. Williams, "An exact dynamic stiffness matrix for coupled extensional-torsional vibration of structural members," Computers and Structures, vol. 50, no. 2, pp. 161-166, 1994.

[9] X. Chen and K. K. Tamma, "Dynamic response of elastic thinwalled structures influenced by coupling effects," Computers and Structures, vol. 51, no. 1, pp. 91-105, 1994.

[10] J. R. Banerjee, S. Guo, and W. P. Howson, "Exact dynamic stiffness matrix of a bending-torsion coupled beam including warping," Computers and Structures, vol. 59, no. 4, pp. 613-621, 1996.

[11] M. Tanaka and A. N. Bercin, "Free vibration solution for uniform beams of nonsymmetrical cross section using Mathematica," Computers and Structures, vol. 71, no. 1, pp. 1-8, 1999.

[12] S. M. Hashemi and M. J. Richard, "A dynamic finite element (DFE) method for free vibrations of bending-torsion coupled beams," Aerospace Science and Technology, vol. 4, no. 1, pp. 4155, 2000.

[13] R. D. Ambrosini, J. D. Riera, and R. F. Danesi, "A modified Vlasov theory for dynamic analysis of thin-walled and variable open section beams," Engineering Structures, vol. 22, no. 8, pp. 890-900, 2000.

[14] L. P. Kollár, "Flexural-torsional vibration of open section composite beams with shear deformation," International Journal of Solids and Structures, vol. 38, no. 42-43, pp. 7543-7558, 2001.

[15] Y. Matsui and T. Hayashikawa, "Dynamic stiffness analysis for torsional vibration of continuous beams with thin-walled crosssection," Journal of Sound and Vibration, vol. 243, no. 2, pp. 301316, 2001.

[16] V. H. Cortínez and M. T. Piovan, "Vibration and buckling of composite thin-walled beams with shear deformability," Journal of Sound and Vibration, vol. 258, no. 4, pp. 701-723, 2002.

[17] A. Arpaci, S. E. Bozdag, and E. Sunbuloglu, "Triply coupled vibrations of thin-walled open cross-section beams including rotary inertia effects," Journal of Sound and Vibration, vol. 260, no. 5, pp. 889-900, 2003. 
[18] M. Y. Kim, H. T. Yun, and N. I. Kim, "Exact dynamic and static element stiffness matrices of nonsymmetric thin-walled beamcolumns," Computers and Structures, vol. 81, no. 14, pp. 14251448, 2003.

[19] L. Jun, L. Wanyou, S. Rongying, and H. Hongxing, "Coupled bending and torsional vibration of nonsymmetrical axially loaded thin-walled Bernoulli-Euler beams," Mechanics Research Communications, vol. 31, no. 6, pp. 697-711, 2004.

[20] L. Jun, H. Hongxing, S. Rongying, and J. Xianding, "Dynamic response of axially loaded monosymmetrical thin-walled Bernoulli-Euler beams," Thin-Walled Structures, vol. 42, no. 12, pp. 1689-1707, 2004.

[21] F. Mohri, L. Azrar, and M. Potier-Ferry, "Vibration analysis of buckled thin-walled beams with open sections," Journal of Sound and Vibration, vol. 275, no. 1-2, pp. 434-446, 2004.

[22] A. Prokić, "On triply coupled vibrations of thin-walled beams with arbitrary cross-section," Journal of Sound and Vibration, vol. 279, no. 3-5, pp. 723-737, 2005.

[23] M. O. Kaya and O. O. Ozgumus, "Flexural-torsional-coupled vibration analysis of axially loaded closed-section composite Timoshenko beam by using DTM," Journal of Sound and Vibration, vol. 306, no. 3-5, pp. 495-506, 2007.

[24] B. Rafezy and W. P. Howson, "Exact dynamic stiffness matrix for a thin-walled beam of doubly asymmetric cross-section filled with shear sensitive material," International Journal for Numerical Methods in Engineering, vol. 69, no. 13, pp. 27582779, 2007.

[25] H. H. Chen and K. M. Hsiao, "Coupled axial-torsional vibration of thin-walled Z-section beam induced by boundary conditions," Thin-Walled Structures, vol. 45, no. 6, pp. 573-583, 2007.

[26] D. Ambrosini, "On free vibration of nonsymmetrical thinwalled beams," Thin-Walled Structures, vol. 47, no. 6-7, pp. 629636, 2009.

[27] G. M. Vörös, "On coupled bending-torsional vibrations of beams with initial loads," Mechanics Research Communications, vol. 36, no. 5, pp. 603-611, 2009.

[28] B. Rafezy and W. P. Howson, "Exact natural frequencies of a three-dimensional shear-torsion beam with doubly asymmetric cross-section using a two-dimensional approach," Journal of Sound and Vibration, vol. 295, no. 3-5, pp. 1044-1059, 2006.

[29] F. de Borbón and D. Ambrosini, "On free vibration analysis of thin-walled beams axially loaded," Thin-Walled Structures, vol. 48, no. 12, pp. 915-920, 2010. 


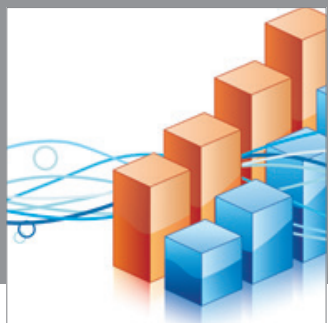

Advances in

Operations Research

mansans

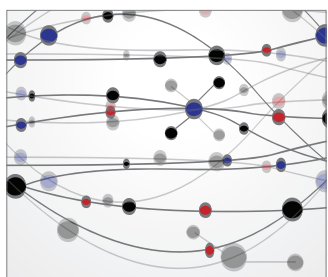

The Scientific World Journal
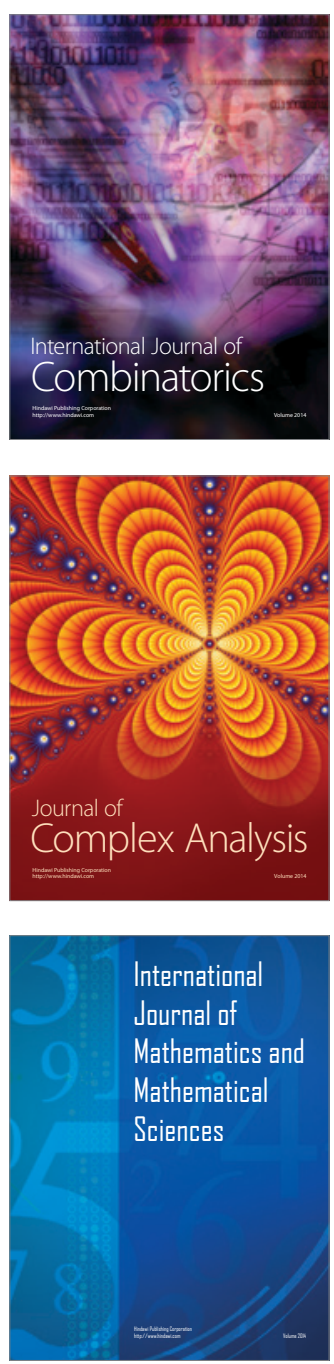
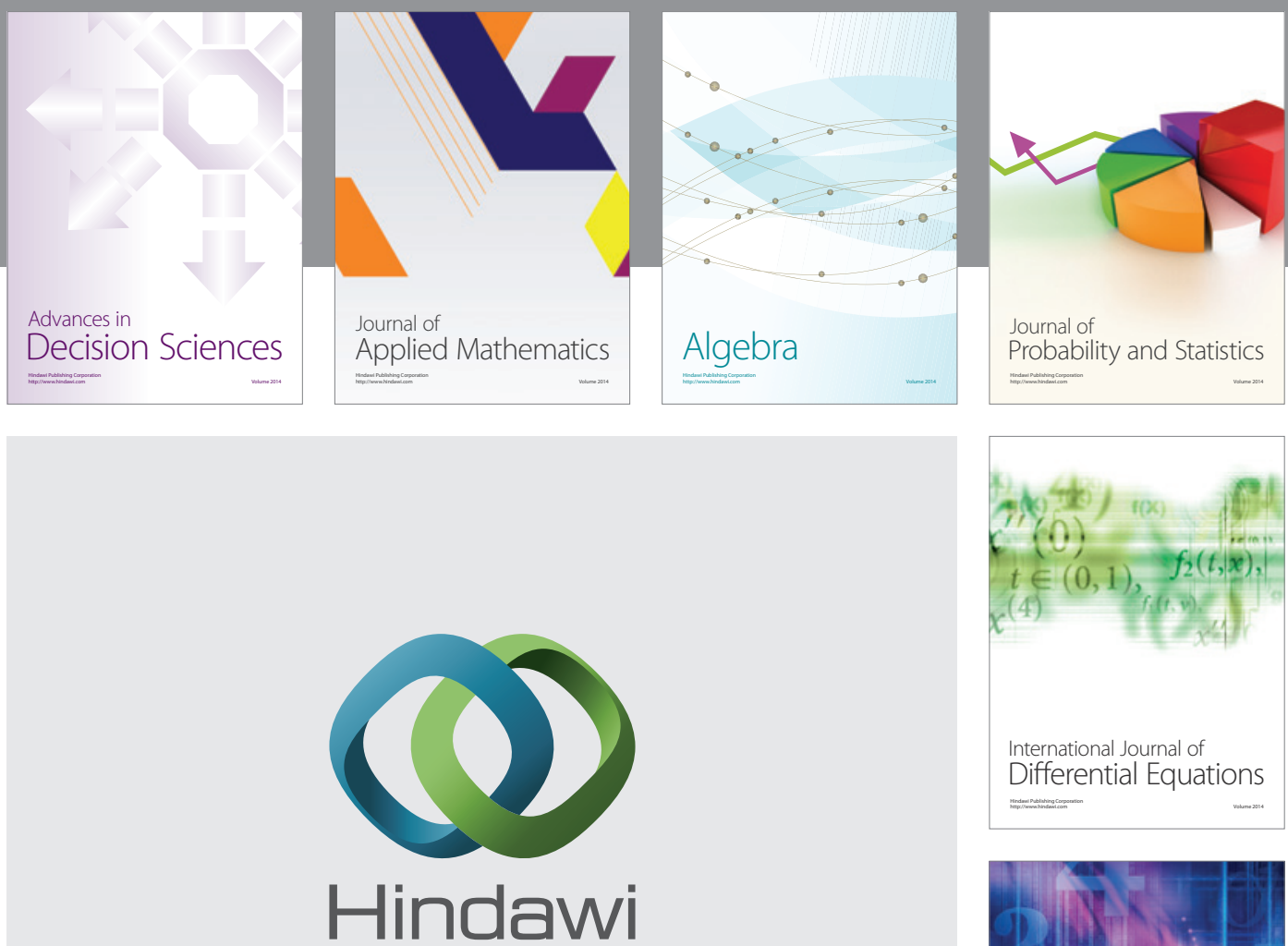

Submit your manuscripts at http://www.hindawi.com
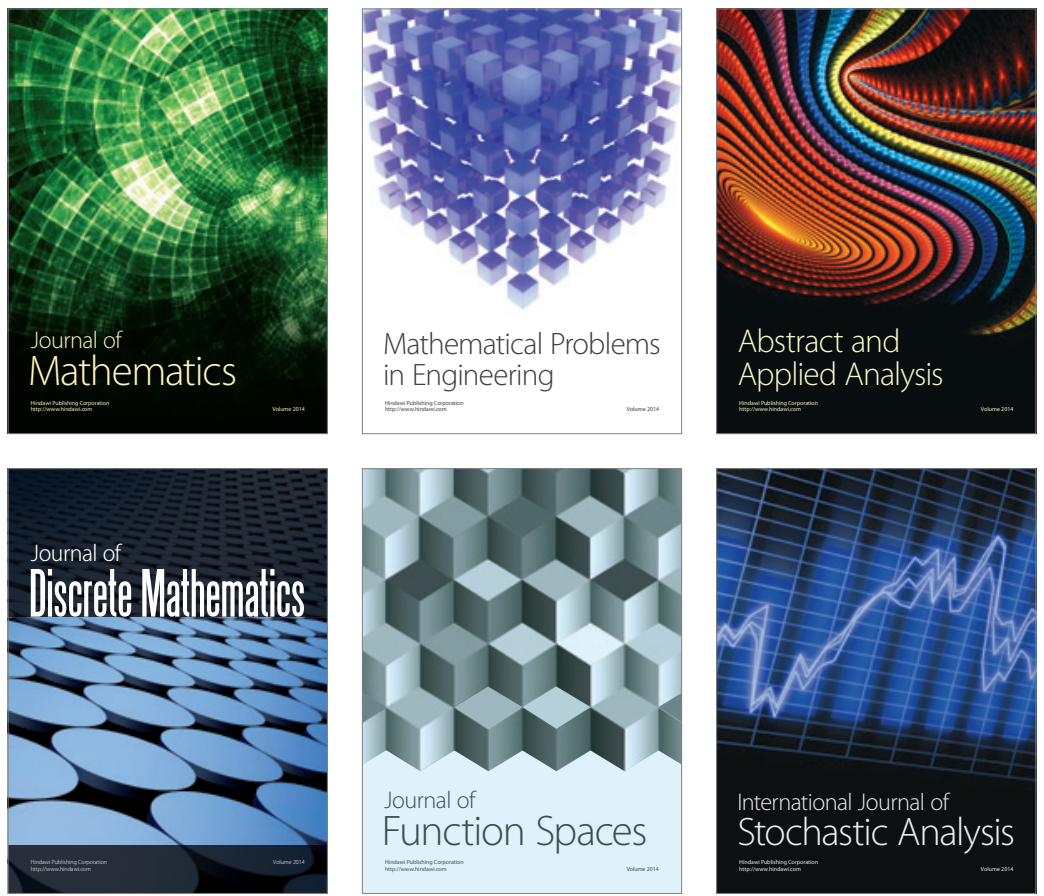

Journal of

Function Spaces

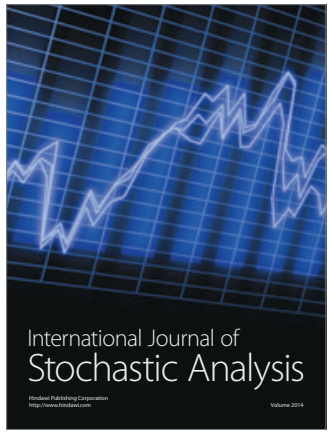

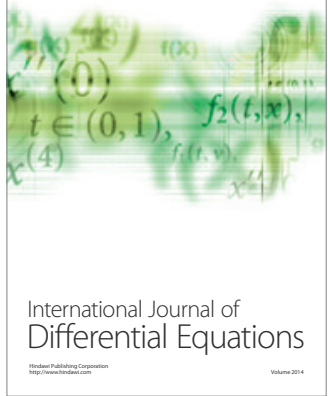
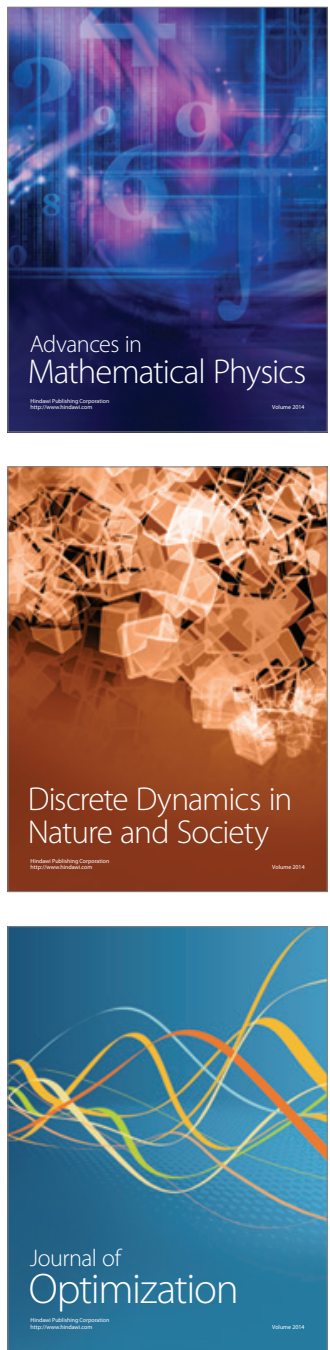